\title{
CARACTERIZAÇÃO FISICO-QUÍMICA E SENSORIAL DE GELEIA DE MAMÃO COM ARAÇÁ-BOI ${ }^{1}$
}

\author{
ELISETH DE SOUZA VIANA², JACIENE LOPES DE JESUS ${ }^{3}$, RONIELLI CARDOSO REIS ${ }^{4}$, \\ MERCIA DAMASCENO FONSECA ${ }^{5}$, CELIO KERSUL DO SACRAMENTO ${ }^{6}$
}

RESUMO - Foram elaboradas quatro formulações de geleia com as seguintes proporções de mamão (Carica papaya) (M) e araçá-boi (Eugenia stipitata) (AB): F1 (70\% M: 30\% AB), F2 (60\% M: 40\% AB), $\mathrm{F} 3(40 \% \mathrm{M}: 60 \% \mathrm{AB})$ e $\mathrm{F} 4(30 \% \mathrm{M}: 70 \% \mathrm{AB})$. Todas as formulações apresentaram proporção final de polpa e açúcar de 1:1, com $0,5 \%$ de pectina e teor de $65 \%$ de sólidos solúveis. As geleias foram analisadas quanto às características físico-químicas e sensoriais (teste de aceitação e preferência). O teste de aceitação foi realizado por 50 consumidores, por meio de escala hedônica de 7 pontos, avaliando-se os atributos: aparência, cor, aroma, sabor e textura. A acidez titulável variou entre $0,77 \mathrm{e} 1,72 \mathrm{~g}$ de ácido cítrico $100 \mathrm{~g}^{-1}$, estando dentro da faixa de acidez determinada para diferentes geleias de frutas. $\mathrm{O}$ teor de açúcares redutores foi mais elevado na formulação F4. As formulações apresentaram índice de aceitação acima de $90 \%$ para os atributos aparência, cor, aroma e textura. As formulações F1 e F2, com menores concentrações de araçá-boi, apresentaram maior aceitação para os atributos sensoriais cor e sabor. Entretanto, a formulação F1 apresentou elevada intenção de compra e foi considerada pelo mapa de preferência interno a mais aceita para todos os atributos avaliados, sendo, portanto, a mais adequada para a industrialização e consumo.

Termos para indexação: Carica papaya, Eugenia stipitata, teste de aceitação, mapa de preferência.

\section{PHYSICOCHEMICAL AND SENSORIAL CHARACTERIZATION OF PAPAYA AND ARAÇA BOI JELLY}

\begin{abstract}
Four formulations of jelly were prepared with the following proportions of papaya (Carica papaya) (P) and araçá-boi (Eugenia stipitata) (AB): F1 (70\% P: 30\% AB), F2 (60\% P: 40\% AB), F3 (40\% P: $60 \% \mathrm{AB})$ and $\mathrm{F} 4(30 \% \mathrm{P}: 70 \% \mathrm{AB})$. Each formulation showed a final proportion of pulp and sugar of $1: 1,0.5 \%$ of pectin and $65^{\circ}$ Brix of soluble solids content. The jellies were analyzed by the physicochemical and sensorial characteristics (acceptance and preference). The sensorial acceptance was performed by 50 consumers using a 7-point hedonic scale, evaluating the following attributes: general appearance, color, aroma, flavor and texture. The titratable acidity presented variation from 0.77 to 1.72 citric acid $100 \mathrm{~g}^{-1}$. These values are in according to the values recommended to different fruit jellies. The greatest content of reducing sugar was found in the F4 formulation. The formulations presented acceptance index of $90 \%$ for the appearance, color, flavor and texture, attributes. The F1 and F2 formulations, which contain less araçá boi pulp content, presented the greater acceptance index to the color and flavor attributes; however, according to the preference mapping methodology the $\mathrm{F} 1$ formulation was pointed as the most acceptable for all the attributes evaluated and it presented high purchase intent score by the consumers, being indicated for the processing and consumption.
\end{abstract}

Index terms: Carica papaya, Eugenia stipitata, acceptance test, preference mapping.

\footnotetext{
1'(Trabalho 016-12). Recebido em: 03-01-2012. Aceito para publicação em: 16-10-2012.

${ }^{2}$ Economista Doméstica, D.Sc. Microbiologia Agrícola, pesquisadora da Embrapa Mandioca e Fruticultura, Rua Embrapa, s/n, CEP 44380-000, Cruz das Almas-BA. E-mail: eliseth.viana@embrapa.br.

${ }^{3}$ Engenheira de Alimentos, Mestranda em Engenharia Química - UFBA, Analista da Embrapa Mandioca e Fruticultura Tropical, Cruz das Almas-BA. E-mail: jaciene.jesus@embrapa.br.

${ }^{4}$ Engenheira de Alimentos, D.Sc. Ciência e Tecnologia de Alimentos, Pesquisadora da Embrapa Mandioca e Fruticultura. Cruz das Almas. E-mail: ronielli.reis@embrapa.br

${ }^{5}$ Graduanda em Farmácia, FAMAM, Cruz das Almas-BA. E-mail: mercia.fonseca@gmail.com

${ }^{6}$ Engenheiro Agrônomo, D.Sc. Produção Vegetal, Professor Pleno da Universidade Estadual de Santa Cruz, Ilhéus - BA. E-mail: celiokersul@gmail.com
} 


\section{INTRODUÇÃO}

O desenvolvimento de novos produtos com elevadas proporções de frutas em suas formulações e com boas propriedades funcionais e nutricionais contribui para diversificar as possibilidades de mercado, principalmente se os produtos forem atrativos, práticos e com maior vida de prateleira (MARTÍNESPARZA et al., $2011 \mathrm{a} \mathrm{e} \mathrm{b).} \mathrm{A} \mathrm{diversidade} \mathrm{das} \mathrm{frutas}$ existentes no território brasileiro com propriedades adequadas para o processamento demonstra que este é um mercado que tem potencial para crescimento (LAGO et al., 2006; MOTA, 2006; YUYAMA et al., 2008; LAGO-VANZELA et al., 2011).

A geleia é um produto de umidade intermediária preparada com polpa de frutas, açúcar, pectina, ácido e outros ingredientes, que permitem sua conservação por um período prolongado (BASU et al., 2011), possibilitando, inclusive, a mistura de frutas para criação de novos sabores.

As geleias mistas associam características de duas ou mais frutas, permitindo a obtenção de produtos com maior valor nutricional e propriedades sensoriais agradáveis, agregando valor e criando possibilidades de conquistar maior espaço junto ao mercado consumidor. Algumas pesquisas têm sido realizadas neste sentido. Ferreira et al. (2010) realizaram estudo com geleias mistas de melancia com tamarindo e obtiveram geleias com elevada aceitação sensorial. Singh et al. (2009) avaliaram a aceitação de diferentes geleias mistas de frutas e verificaram maior aceitação para a geleia de mamão com abacaxi, indicando o potencial do mamão na elaboração destes produtos.

Desse modo, com a enorme variedade de frutas produzidas no Brasil, surge a possibilidade de associação de sabores, para confecção de geleias mistas, com a utilização de frutas já cultivadas, como o mamão, e frutas potenciais, mas ainda pouco conhecidas, como o araçá-boi.

O mamoeiro (Carica papaya) é uma fruteira presente em quase todos os países da América Tropical, e seus frutos são excelentes fontes de cálcio, pró-vitamina A e vitamina $\mathrm{C}$, sendo por isso amplamente utilizados em dietas alimentares (SERRANO; CATTANEO, 2010).

$\mathrm{O}$ araçá-boi (Eugenia stipitata McVaugh) é uma fruta nativa da Amazônia Ocidental e das Guianas, sendo adaptada aos climas tropicais e subtropicais. No Brasil, é encontrado na região Amazônica, Mato Grosso e Bahia, mas ainda sem exploração comercial. O fruto é uma baga globosa, com casca fina, cor amarelo-canário quando maduro, e aveludada, pesando de 30 a $800 \mathrm{~g}$, apresentando formato arredondado ou achatado, com diâmetro longitudinal de 5 a $10 \mathrm{~cm}$ e transversal de 5 a 12 $\mathrm{cm}$. Sua polpa é suculenta, ácida, de coloração amarelo-clara, pouco fibrosa, possui de 4 a 10 sementes oblongas, medindo 0,5 a $1,0 \mathrm{~cm}$ de comprimento (SACRAMENTO et al., 2008).

Os frutos de araçá-boi apresentam teor de sólidos solúveis de $5,54{ }^{\circ} \mathrm{Brix}$, valor inferior à maioria dos frutos tropicais, e acidez igual a 2,38 $\mathrm{g}$ de ácido cítrico $100 \mathrm{~g}^{-1}$, valor superior à acidez das frutas utilizadas para a agroindustrialização. Assim, a relação brix/acidez é extremamente baixa $(2,33)$, o que prejudica a aceitação dos frutos pelos consumidores brasileiros. Entretanto, a polpa de araçá-boi adicionada de açúcar tem sido utilizada com sucesso na fabricação de sorvetes, e apresenta potencial para o uso em formulações de sucos, geleias e néctares, especialmente quando associada às frutas de baixa acidez, como manga, mamão e maçã (SACRAMENTO et al., 2008).

O sucesso de um alimento no mercado depende de seu desempenho junto ao consumidor. No processo de desenvolvimento de novos produtos, a determinação da aceitação e/ou preferência do produto torna- se indispensável, e, neste aspecto, os testes sensoriais mais empregados para obter informações sobre a aceitação de um novo produto são os testes afetivos de aceitação e de preferência (STONE; SIDEL, 2004). Entretanto, as metodologias tradicionais para analisar testes de aceitação têm mostrado limitações e deficiências, uma vez que os produtos são comparados por análise de variância e testes de comparação de médias. Dessa forma, para cada produto avaliado, obtém-se a média do grupo de consumidores, assumindo, portanto, que todos os consumidores possuem o mesmo comportamento, sem considerar suas individualidades. Com isto, pode estar ocorrendo perda de importantes informações sobre os produtos avaliados .

O mapa de preferência interno (MDPREF) baseia-se em métodos estatísticos multivariados, sendo a Análise de Componentes Principais (ACP) um dos mais usados. É um procedimento estatístico multidimensional que considera a individualidade dos consumidores e não somente a média do grupo que avaliou os produtos. É uma representação gráfica das diferenças de aceitação entre as amostras, a qual permite a identificação de cada consumidor e suas preferências em relação às amostras avaliadas.

Com base no exposto, este trabalho teve como objetivos desenvolver diferentes formulações de geleias mistas de mamão e araçá-boi, e avaliar a qualidade sensorial dos produtos obtidos, na tentativa de obter um produto com elevada aceitação sensorial. 


\section{MATERIAL E MÉTODOS}

\section{Obtenção dos frutos}

Foram utilizados mamões 'Sunrise Solo', produzidos na Fazenda Palmares, município de Porto Seguro-BA. Os araçás-bois foram coletados na Fazenda Ouro Verde, distrito de Vila Brasil, município de Uma-BA.

Processamento da geleia mista de mamão e araçá-boi

Os frutos foram transportados para o laboratório de Ciência e Tecnologia de Alimentos da Embrapa, onde foram selecionados para tornar o lote mais uniforme quanto ao estádio de maturação e eliminar aqueles que apresentavam injúrias mecânicas ou podridões. Em seguida, os frutos selecionados foram lavados e sanitizados em solução de hipoclorito de sódio contendo cloro ativo $\left(50 \mathrm{mg} \mathrm{L}^{-1}\right)$, por 15 minutos. A polpa de araçá-boi foi obtida por meio de despolpadeira semi-industrial (Tecnint), e para obtenção da polpa de mamão utilizou-se liquidificador doméstico.

As geleias foram elaboradas segundo metodologia proposta por Jackix (1988), com modificações. Diferentes proporções de polpas de mamão $(\mathrm{M})$ e araçá-boi (AB) foram adicionadas de sacarose, na proporção polpa e sacarose $60: 40$, e pectina cítrica 105 CP Kelco (CP Kelco do Brasil), na concentração de $0,5 \%(\mathrm{~m} / \mathrm{m})$. Quatro formulações de geleias foram desenvolvidas: F1 (70\% M: 30\% AB:), F2 (60\% M: $40 \%$ AB), F3 (40\% M: $60 \%$ AB) e F4 $(30 \% \mathrm{M}$ : $70 \% \mathrm{AB})$.

Após a mistura da polpa e de $75 \%$ do total de sacarose, mediu-se o teor de sólidos solúveis e o $\mathrm{pH}$, fazendo-se os ajustes necessários (com adição de ácido ou base) para obter o $\mathrm{pH}$ próximo a 3,0, que é ideal para elaboração de geleias . Em seguida, procedeu-se à cocção em tacho aberto com agitação manual contínua. A adição da pectina constitui etapa crítica no processo de fabricação de geleias, por isto foi acrescentada somente ao final do processo de cocção, para que a mesma não fosse degradada por aquecimento excessivo. Para dissolver a pectina, a mesma foi misturada com o restante de sacarose ( $25 \%$ do total) e com $150 \mathrm{~mL}$ de água a $60{ }^{\circ} \mathrm{C}$, sob agitação constante. Quando a geleia atingiu $63^{\circ}$ Brix, adicionou-se a pectina diluída e misturou-se vigorosamente a fim de incorporá-la completamente ao produto.

Após atingir $65^{\circ}$ Brix, a geleia foi envasada em frascos de vidro de $250 \mathrm{~g}$, sob temperaturas próximas a $94{ }^{\circ} \mathrm{C}$. Deixou-se um espaço livre de aproximadamente um centímetro em cada embalagem a fim de favorecer a formação do vácuo. A seguir, os recipientes foram hermeticamente fechados.

O resfriamento do produto foi feito por meio da imersão dos vidros de geleia em água fria, até que se atingisse temperatura inferior a $40^{\circ} \mathrm{C}$. As embalagens secas foram rotuladas e mantidas à temperatura ambiente.

\section{Análises físico-químicas das polpas e das geleias}

As polpas de mamão e araçá-boi e as geleias mistas foram analisadas quanto ao teor de sólidos solúveis (SS) expressos em ${ }^{\circ} \mathrm{Brix}, \mathrm{pH}$, acidez titulável (AT), expressa em g ácido cítrico $100 \mathrm{~g}^{-1}, \mathrm{e}$ relação SS/AT (ratio), de acordo com a metodologia preconizada pelo Instituto Adolfo Lutz -IAL (2005).

As geleias mistas também foram analisadas quanto ao teor de cinzas e umidade, seguindo-se a metodologia prescrita pelo IAL (2005). Os açúcares redutores e os açúcares totais foram determinados por espectrofotometria (NELSON, 1944; SOMOGYI, 1945), sendo a etapa da hidrólise ácida realizada segundo o IAL (2005).

Todas as análises foram realizadas em triplicata.

\section{Análise Sensorial}

\section{Teste de aceitação e intenção de compra}

$\mathrm{O}$ teste de aceitação sensorial foi conduzido em cabines individuais, com iluminação branca, com a participação de cinquenta julgadores não treinados. As amostras foram oferecidas aos provadores em copos plásticos descartáveis de $50 \mathrm{~mL}$, codificados com números aleatórios de três dígitos, à temperatura ambiente, acompanhadas de biscoitos "cream cracker" e água. Foram avaliados os atributos sensoriais aparência, cor, aroma, sabor e textura, por meio de uma escala hedônica estruturada de sete pontos, com os termos "desgostei muito" (1) e "gostei muito" (7) nos extremos da escala.

Para avaliar a atitude do consumidor numa situação hipotética de compra do produto, foi utilizada uma escala de intenção de compra de 5 categorias, com os termos "certamente não compraria" (1) e "certamente compraria" (5) nos extremos da escala.

\section{Teste de Preferência}

Foi realizado o teste de ordenação-preferência, e para a amostra (geleia) "mais preferida" atribuiu-se a ordem 1 e para a "menos preferida" a ordem 4 .

Delineamento experimental e análise esta-

\section{tística dos resultados}

O experimento foi conduzido no delineamento inteiramente casualizado, com três repetições. Os dados do teste de aceitação foram submetidos à análise de variância (ANOVA), comparação entre 
as médias pelo teste de Tukey,a $5 \%$ de probabilidade, análise de frequência, análise de componentes principais para a obtenção do Mapa de Preferência Interno e agrupamento. A análise de frequência dos escores hedônicos para cada amostra de geleia foi realizada de acordo com as seguintes faixas de aceitação:

Rejeição: escores de 1 a 3 indicam que os consumidores desgostaram da amostra.

Aceitação: escores de 4 a 7 indicam que os consumidores gostaram da amostra.

O Mapa de Preferência Interno (MDPREF) foi obtido empregando-se a técnica de Análise de Componentes Principais. Os dados do teste de aceitação foram organizados em uma matriz com os tratamentos (formulações) nas linhas e os consumidores nas colunas, e então submetidos à Análise de Componentes Principais (ACP) a partir da matriz de covariâncias. Todas as análises foram realizadas, utilizando-se do programa Statistica 7.Foi realizado o agrupamento das diferentes formulações de geleia, utilizando todos os dados de aceitação, independentemente do atributo sensorial avaliado, utilizando-se do coeficiente de similaridade e o método UPGMA (Unweighted Pair-Group Method with Arithmetical Average), utilizando-se do programa Statistica 7.0.

\section{RESULTADOS E DISCUSSÃO}

\section{Caracterização físico-química das polpas e das geleias mistas}

A polpa de araçá-boi apresentou baixos teores de $\mathrm{SS}$ e $\mathrm{pH}$, quando comparada com a polpa de mamão (Tabela 1). A acidez da polpa de araçá-boi foi aproximadamente 26 vezes superior à do mamão, e tais características físico-químicas das polpas demonstram que esses frutos associados são uma excelente combinação para o processamento de geleias.

O teor de umidade das quatro formulações variou de $25,99 \%$ (F4) a $29,93 \%$ (F3), semelhante aos encontrados nos trabalhos de Lago et al. (2006) e Lago-Vanzela et al. (2011) em geleia de jambolão $(29,6 \%)$ e cajá-manga $(29,5 \%)$, respectivamente. Mota (2006) verificou teores de umidade mais elevados $(42,84$ a $46,44 \%)$ em geleias produzidas a partir de diferentes variedades de amora-preta. É importante destacar que o teor de umidade está diretamente relacionado com a conservação do produto durante $\mathrm{o}$ armazenamento.

A AT das diferentes formulações variou entre 0,73 e $1,73 \mathrm{~g}$ ácido cítrico $100 \mathrm{~g}^{-1}$, e está de acordo com a faixa de acidez mencionada em outros trabalhos sobre diferentes geleias de frutas (MOTA,
2006; MOTA, 2007; SINGH et al., 2009; LAGO VANZELA et al., 2011). Os autores atribuíram as variações encontradas às diferenças nos teores de ácidos das polpas e suas respectivas proporções utilizadas nas formulações. Singh et al. (2009) verificaram acidez de $1,04 \%$ e $0,83 \%$ em geleias mistas de abacaxi com mamão e laranja com mamão, respectivamente. Neste estudo, a formulação F4 apresentou menor $\mathrm{pH}(3,07)$ e maior acidez $\left(1,73 \mathrm{~g}\right.$ ácido cítrico $\left.100 \mathrm{~g}^{-1}\right)$ devido à maior proporção de polpa de araçá-boi utilizada nessa formulação. Observa-se claramente uma redução do $\mathrm{pH}$ com consequente aumento da acidez, à medida que maiores teores de polpa de araçá-boi foram adicionados nas formulações. Resultado semelhante foi encontrado por Ferreira et al. (2010) em geleias mistas de melancia e tamarindo, em que a adição de tamarindo promoveu aumento da acidez da geleia com consequente redução do $\mathrm{pH}$.

$\mathrm{O}$ teor de açúcares redutores foi mais elevado na formulação F4 (50,85\%), diferindo significativamente $(p \leq 0,05)$ das demais. Esse resultado pode ser atribuído à elevada acidez que favoreceu a hidrólise da sacarose. Lago-Vanzela et al. (2011) também constataram elevada hidrólise dos açúcares não redutores em geleias elaboradas com cajá-manga, atribuindo tal efeito à acidez da fruta.

O teor de sólidos solúveis das formulações variou entre $63,9^{\circ} \mathrm{Brix}$ (F2) e $65,4^{\circ} \mathrm{Brix}$ (F3). Valor semelhante foi verificado por Mota (2007), em geleia de amora-preta $\left(65,64^{\circ} \mathrm{Brix}\right)$. Valores mais elevados de sólidos solúveis foram obtidos por Lago-Vanzela et al. (2011) em geleias de manga $\left(69^{\circ}\right.$ Brix) e por Singh et al. (2009) em geleias mistas de abacaxi com mamão $\left(70,5^{\circ}\right.$ Brix) e mamão com laranja (72,5 ${ }^{\circ}$ Brix). Em estudo realizado por Mota (2006), as geleias formuladas com diferentes variedades de amora-preta apresentaram menores teores de sólidos solúveis (47 a $58^{\circ}$ Brix), quando comparados com os resultados obtidos no presente trabalho. O teor de sólidos solúveis ideal para geleias é de $67,5^{\circ}$ Brix, sendo que, para valores menores (64 $\left.{ }^{\circ} \mathrm{Brix}\right)$, o gel torna-se mais fraco, e acima $\left(71^{\circ} \mathrm{Brix}\right)$ pode ocorrer a cristalização da geleia.

\section{Aceitação Sensorial}

As médias dos escores de aceitação para as quatro formulações de geleia estão apresentadas na Tabela 2. Houve diferença significativa $(p<0,05)$ entre as quatro formulações para cor e sabor, indicando que tais atributos são responsáveis pela diferenciação entre as amostras de geleia.

As formulações F1 e F2, que possuem menores concentrações de araçá-boi, apresentaram maior aceitação para os atributos sensoriais cor e sabor, estando entre os termos hedônicos "gostei modera- 
damente" e "gostei muito" para o sabor, e entre os termos hedônicos "gostei muito" e "gostei muitíssimo" para o atributo cor (Tabela 2).

As frequências de aceitação e rejeição para as formulações de geleia para cada atributo estão apresentadas na Figura 1. Observa-se que todas as formulações apresentaram índice de aceitação acima de $90 \%$ para os atributos aparência, cor, aroma e textura. Para o atributo sabor, as formulações F1 e F2 apresentaram apenas 4\% de rejeição, enquanto F3 e F4 foram as de maior rejeição, com índices de 16 e $28 \%$, respectivamente. Nota-se que a adição de araçá-boi contribui positivamente na aceitação da geleia para os atributos aparência, cor, aroma e textura, e negativamente para o sabor. Esse fato pode ser atribuído à elevada acidez do araçá-boi nas formulações F3 e F4, tornando a geleia com gosto mais ácido, que provavelmente desagradou os consumidores.

A partir da análise de agrupamento realizada, desconsiderando os atributos individualmente, verifica-se a formação de dois grupos, sendo o primeiro formado pelas formulações F2, F3 e F4, e o segundo formado pela formulação F1 (Figura 2). Esse resultado indica que os consumidores, de uma forma global, diferenciaram as amostras considerando a formulação F1 diferente das demais.

O resultado do teste de ordenação-preferência está apresentado na Tabela 3. Valores com as menores somas das ordens indicam que tais amostras são mais preferidas pelos consumidores uma vez que se utilizou escala decrescente de preferência. As Formulações F1 e F2 não diferiram entre si ( $\mathrm{p} \geq 0,05)$, indicando que estas apresentaram maior preferência (menor soma das ordens) pelos consumidores. As formulações F3 e F4 não diferiram significativamente entre si $(p \geq 0,05)$ e apresentaram as maiores somas das ordens, sendo, portanto, consideradas as de menor preferência. Os resultados do teste de preferência reforçam os já obtidos pelo teste de aceitação (Tabela 2), uma vez que a preferência das geleias decresceu com o aumento do teor de araçá-boi.

Mapa de Preferência Interno da geleia de araçá-boi com mamão

Os mapas de preferência interno, gerados a partir dos dados de aceitação em relação aos atributos aparência, cor, aroma, sabor e textura, para as quatro formulações de geleias, estão apresentados na Figura 3. Os escores (notas de aceitação dadas para cada formulação) determinam a posição de cada formulação em relação aos dois primeiros componentes principais, para cada atributo avaliado. Cada ponto representa a correlação dos dados de aceitação de um consumidor com os dois primeiros componentes principais, ou seja, cada ponto representa um consumidor.
Os consumidores que estão posicionados na região central do gráfico não apresentaram correlações significativas $(\mathrm{p} \geq 0,05)$ com os dois componentes principais; logo, tais consumidores não diferenciaram as amostras em relação aos atributos avaliados.

Para cada atributo avaliado, os dois primeiros componentes são superiores a 70\%, sendo suficientes para representar a dispersão das formulações, explicando a maior parte da variação dos dados. Portanto, tem-se uma apresentação bidimensional de fácil interpretação. $\mathrm{Na}$ Análise de Componentes Principais, a porcentagem de explicação dos dados de aceitação varia de acordo com o número de amostras avaliadas, com a diferença percebida entre as amostras pelos consumidores e com o atributo avaliado. Assim, quando se avalia um número pequeno de amostras,os consumidores diferenciam estas em relação à aceitação, e a porcentagem de explicação que cada componente principal acumulará, será maior. Por outro lado, se o número de amostras avaliadas é muito grande e/ou os consumidores não diferenciam tais amostras, a porcentagem de explicação de cada componente será menor.

Em relação à aparência (Figura $3 \mathrm{~A}$ ), os dois componentes principais explicam $81,5 \%$ da variância dos dados de aceitação. Observa-se que a maioria dos consumidores está situada do lado direito do gráfico (primeiro e quarto quadrantes), indicando claramente a preferência pela formulação F1. Um pequeno grupo de consumidores preferiu as demais formulações. Comparando os resultados do mapa de preferência para tal atributo com os resultados do teste de médias (Tabela 2), percebe-se que, assim como para a cor, houve diferenças entre os resultados. O mapa mostrou claramente que a formulação F1 foi mais aceita do que as demais, enquanto pelo teste de média este fato não ocorreu.

A Figura 3B representa o mapa de preferência para o atributo cor. Os dois componentes principais explicam $81,6 \%$ da variação dos dados, sendo que Y1 explica a maior variação $(57,4 \%)$. As formulações F2 e F4 foram consideradas semelhantes pelos consumidores em relação à cor. Pela posição espacial das amostras, há formação de três grupos distintos e com aceitação diferente. Nota-se que houve concentração dos consumidores no primeiro e quarto quadrantes, indicando maior aceitação da formulação F1. Um grupo pequeno de consumidores correlacionado negativamente com o Y2 atribuiu maiores notas para as geleias F2 e F4. A formulação F3 apresentou menor aceitação. Nota-se que os resultados obtidos pelo mapa de preferência não estão de acordo com os obtidos a partir do teste de média (Tabela 2), em que as formulações F1 e F2 foram 
consideradas com mesma aceitação para o atributo cor. Isto ocorre porque, na análise de componentes principais, considera a opinião de cada consumidor, sendo, portanto uma análise que retrata de forma mais real o que ocorre no processo de aceitação.

A Figura 3C apresenta o mapa de preferência interno para o atributo aroma. Verifica-se que os dois componentes principais explicam $74 \%$ da variância dos dados de aceitação. Observa-se que as formulações F2 e F3, localizadas no terceiro quadrante, estão em posições muito próximas, indicando que estas não diferem entre si quanto à aceitação pelos consumidores. Pela disposição das quatro formulações no gráfico, sugere-se a formação de 3 grupos distintos. Entretanto, os consumidores encontram-se dispersos nos quatro quadrantes, havendo aceitação para as quatro formulações de geleia.

Para o atributo sabor (Figura 3D), o primeiro componente principal (Y1) explica $54,6 \%$ e o segundo componente (Y2) explica $25,5 \%$ da variância de aceitação entre as quatro formulações de geleia. Os dois primeiros componentes explicam $80,1 \%$ da variância total dos dados de aceitação. A separação espacial das quatro formulações sugere a existência de diferença na aceitação entre as geleias em relação ao atributo sabor. A maioria dos consumidores está correlacionada positivamente ao primeiro componente principal (Y1), situados no primeiro e quarto quadrantes, preferindo as formulações F1 e F2, concordando com os resultados obtidos a partir do teste de média (Tabela 2).

Em relação ao atributo textura, Y1 representa $44,4 \%$ da variação dos dados, e Y2 31,7\%, totalizando 76,1 da variância total dos dados de aceitação (Figura 3E). Os consumidores correlacionados positivamente a Y1 e Y2 preferiram a formulação $F 1$, e os consumidores correlacionados positivamente a Y2, e negativamente com Y1, consideraram F3 a de maior aceitação. As formulações F2 e F4 tiveram aceitação por alguns consumidores.

Por meio de uma análise global do mapa de preferência obtido pelos dados de aceitação, nota-se que a formulação $\mathrm{F} 1$, contendo $70 \%$ de mamão e $30 \%$ de araçá-boi, foi preferida pelos consumidores em relação a todos os atributos avaliados. Os resultados revelaram que os consumidores optaram por uma geleia com menor proporção de araçá-boi e maior de mamão.

Quantoà intenção de compra dos consumidores, agrupando-se as categorias "certamente compraria" e "possivelmente compraria", $74 \%$ indicaram que comprariam a formulação F1 $(70 \%$ de mamão e $30 \%$ de araçá-boi), caso essa fosse comercializada (Figura 4). Observa-se que o percentual de intenção de compra da geleia diminuiu à medida que maior concentração de araçá-boi foi utilizada nas formulações. Para a formulação F4, contendo $30 \%$ de mamão e $70 \%$ de araçá-boi, apenas $44 \%$ dos consumidores manifestaram intenção de comprar o produto.

TABELA 1- Médias e desvios-padrão das características físico-químicas das polpas e das geleias de mamão (M) com araçá-boi (AB) em diferentes proporções.

\begin{tabular}{lccccccc}
\hline \multirow{2}{*}{ Parâmetros avaliados } & \multicolumn{3}{c}{ Polpas } & & \multicolumn{4}{c}{ Formulações das geleias } \\
\cline { 2 - 7 } & Mamão & Araçá-boi & F1 & F2 & F3 & F4 \\
\hline Sólidos Solúveis ('Brix) & $10,08 \pm 0,14$ & $4,58 \pm 0,14$ & $65,08 \mathrm{~b}$ & $63,92 \mathrm{c}$ & $65,42 \mathrm{a}$ & $65,25 \mathrm{ab}$ \\
pH & $4,83 \pm 0,02$ & $2,51 \pm 0,11$ & $3,53 \mathrm{a}$ & $3,33 \mathrm{~b}$ & $3,16 \mathrm{c}$ & $3,07 \mathrm{~d}$ \\
Acidez titulável (g ácido cítrico $100 \mathrm{~g}^{-1}$ ) & $0,11 \pm 0,01$ & $2,88 \pm 0,02$ & $0,73 \mathrm{~d}$ & $1,01 \mathrm{c}$ & $1,45 \mathrm{~b}$ & $1,73 \mathrm{a}$ \\
Ratio (SS/AT) & $91,97 \pm 1,31$ & $1,65 \pm 0,04$ & $89,15 \mathrm{a}$ & $63,29 \mathrm{~b}$ & $45,12 \mathrm{c}$ & $37,72 \mathrm{~d}$ \\
Açúcares redutores(\%) & - & - & $31,30 \mathrm{~d}$ & $33,57 \mathrm{c}$ & $41,40 \mathrm{~b}$ & $50,85 \mathrm{a}$ \\
Açúcares totais (\%) & - & - & $53,57 \mathrm{ab}$ & $54,11 \mathrm{ab}$ & $52,76 \mathrm{~b}$ & $58,73 \mathrm{a}$ \\
Cinzas (\%) & - & - & $0,32 \mathrm{a}$ & $0,31 \mathrm{a}$ & $0,26 \mathrm{~b}$ & $0,25 \mathrm{~b}$ \\
Umidade (\%) & - & - & $27,99 \mathrm{~b}$ & $26,49 \mathrm{c}$ & $29,93 \mathrm{a}$ & $25,99 \mathrm{c}$ \\
\hline
\end{tabular}

Médias ( $n=3$ ) seguidas por letras iguais, na linha, não diferem entre si, ao nível de $5 \%$ de significância, pelo teste de Tukey. F1 (70\% $\mathrm{M}: 30 \% \mathrm{AB}:), \mathrm{F} 2(60 \% \mathrm{M}: 40 \% \mathrm{AB}), \mathrm{F} 3(40 \% \mathrm{M}: 60 \% \mathrm{AB})$ e F4 (30\% M : 70\% AB). 
TABELA 2 - Médias dos valores hedônicos obtidos pelo teste de aceitação em relação aos atributos aparência, cor, aroma, sabor e textura das geleias elaboradas com mamão (M) e araçá-boi (AB) em diferentes proporções.

\begin{tabular}{cccccc}
\hline Formulações & Aparência & Cor & Aroma & Sabor & Textura \\
\hline F1 & $5,86 \mathrm{a}$ & $6,40 \mathrm{a}$ & $5,40 \mathrm{a}$ & $6,10 \mathrm{a}$ & $5,88 \mathrm{a}$ \\
F2 & $5,62 \mathrm{a}$ & $6,00 \mathrm{ab}$ & $5,78 \mathrm{a}$ & $5,84 \mathrm{ab}$ & $5,94 \mathrm{a}$ \\
F3 & $5,56 \mathrm{a}$ & $5,74 \mathrm{~b}$ & $5,64 \mathrm{a}$ & $5,22 \mathrm{bc}$ & $5,86 \mathrm{a}$ \\
F4 & $5,64 \mathrm{a}$ & $5,90 \mathrm{~b}$ & $5,54 \mathrm{a}$ & $4,86 \mathrm{c}$ & $5,72 \mathrm{a}$ \\
\hline
\end{tabular}

Médias ( $\mathrm{n}=50$ ) seguidas por letras iguais, na coluna, não diferem entre si, ao nível de 5\% de significância, pelo teste de Tukey. F1 (70\% M: 30\% AB:), F2 (60\% M: 40\% AB), F3 (40\%M: 60\% AB) e F4 (30\% M: $70 \% \mathrm{AB})$.

Aparèncla

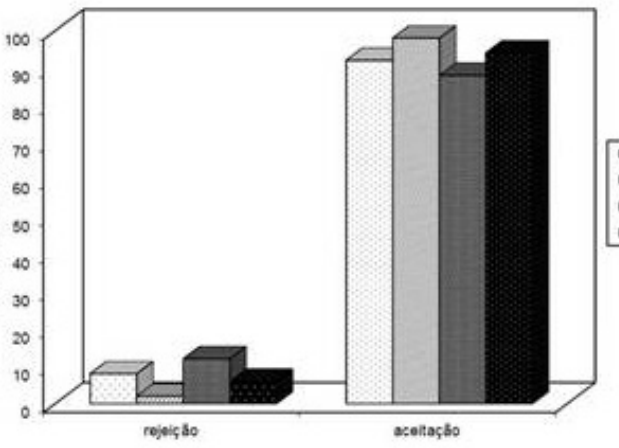

Aroma

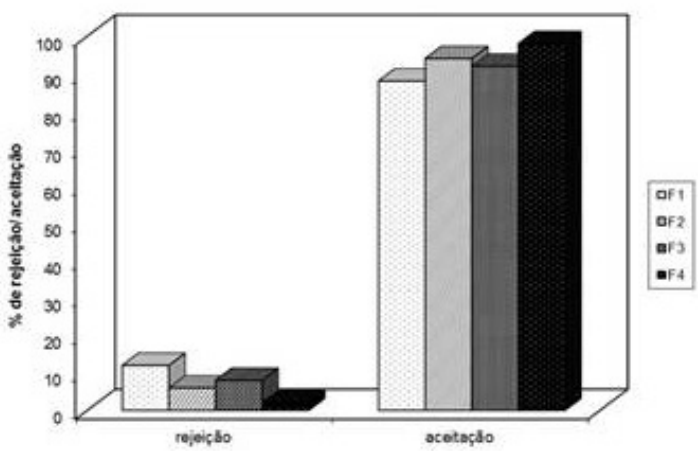

Cor

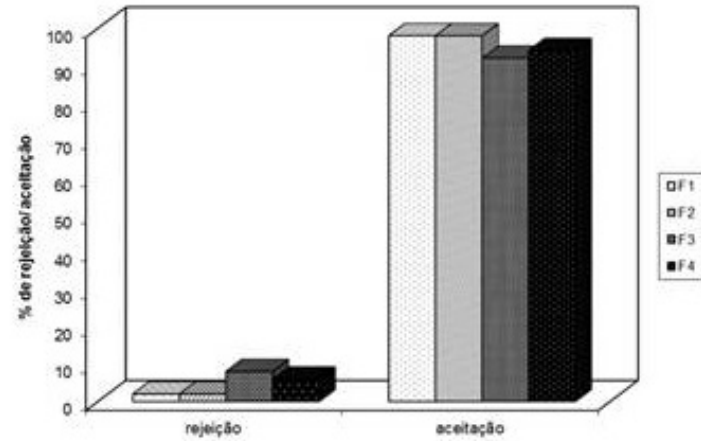

Sabor

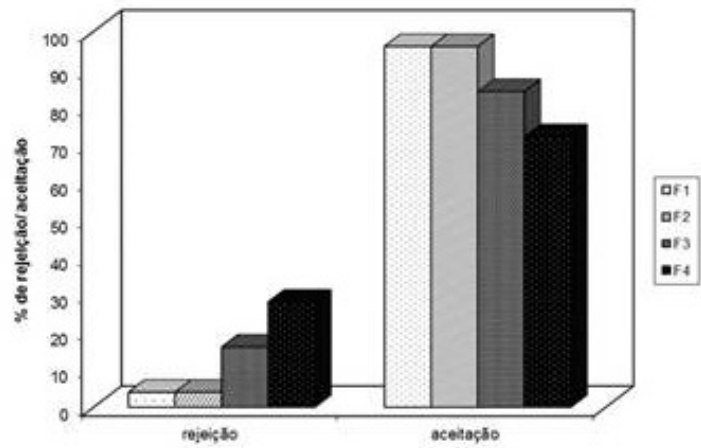

Textura

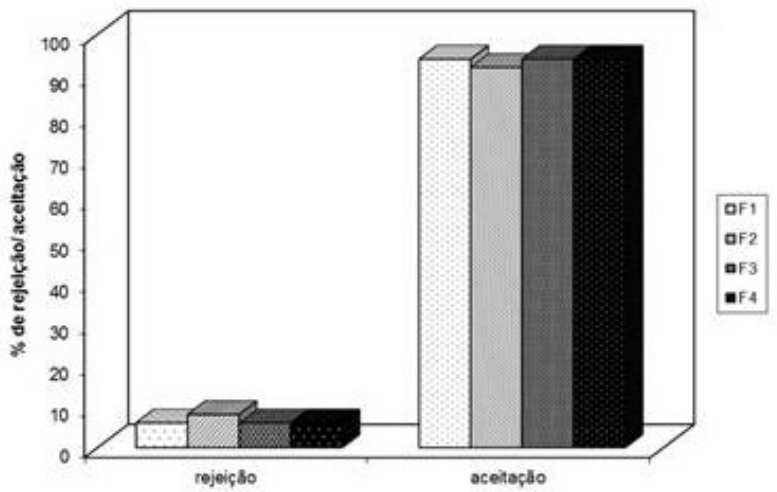

FIGURA 1- Frequências de aceitação e rejeição pelos consumidores para as quatro formulações de geleia de mamão $(\mathrm{M})$ e araçá-boi $(\mathrm{AB})$ referentes a cada atributo sensorial avaliado. F1 (70\% M: $30 \% \mathrm{AB}), \mathrm{F} 2(60 \% \mathrm{M}: 40 \% \mathrm{AB}), \mathrm{F} 3(40 \% \mathrm{M}: 60 \% \mathrm{AB})$ e F4 (30\% M: 70\% AB). 


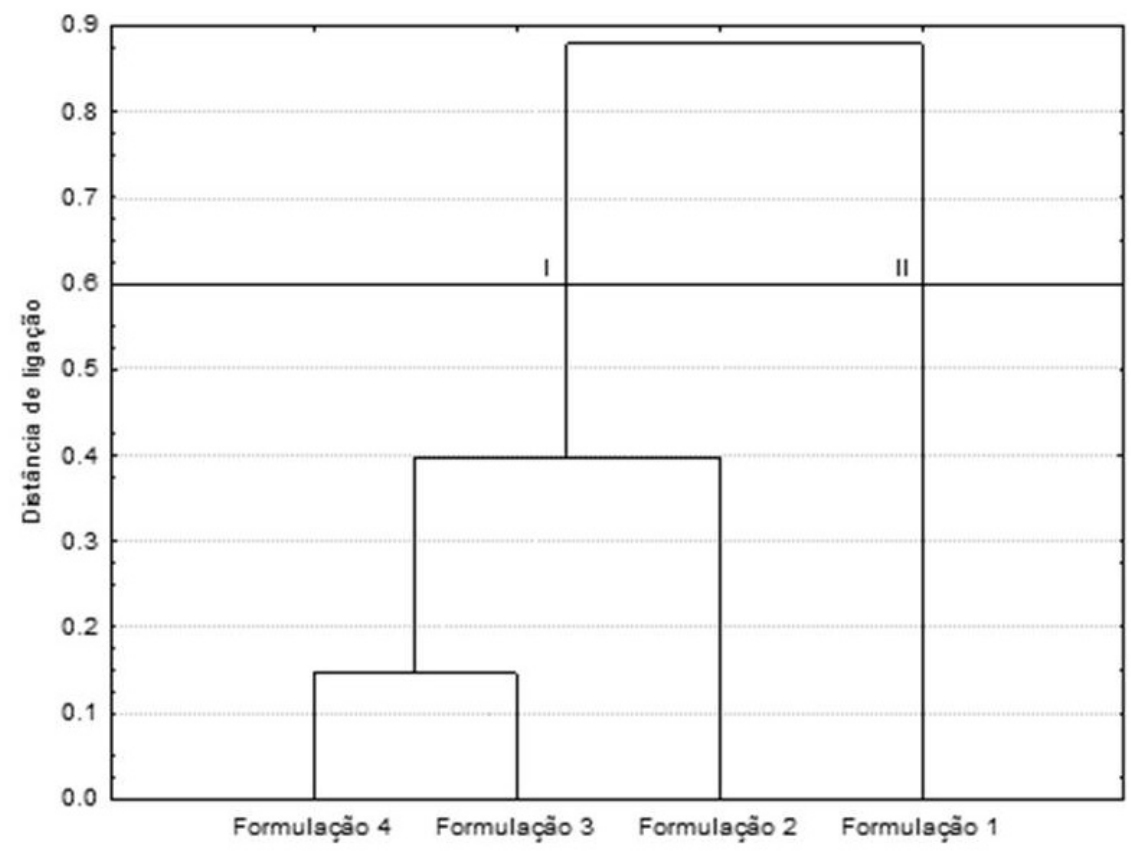

FIGURA 2- Análise de agrupamentos do teste de aceitação das geleias formuladas com diferentes proporções de mamão (M) e araçá-boi (AB). F1 (70\% M: 30\% AB), F2 (60\% M: 40\% AB), F3 (40\% M: $60 \% \mathrm{AB})$ e $\mathrm{F} 4(30 \% \mathrm{M}: 70 \% \mathrm{AB})$.

TABELA 3 - Diferenças entre as somas das ordens das quatro formulações de geleia de mamão (M) com araçá-boi $(\mathrm{AB})$.

\begin{tabular}{|c|c|c|c|c|}
\hline \multirow{3}{*}{ Soma total } & \multicolumn{4}{|c|}{ Diferença entre a soma das ordens de cada amostra } \\
\hline & F1 & F2 & F3 & F4 \\
\hline & $104 \mathrm{a}$ & $112 \mathrm{a}$ & $128 \mathrm{ab}$ & $157 \mathrm{~b}$ \\
\hline F1 & - & $8^{\mathrm{ns}}$ & $24^{\mathrm{ns}}$ & $53 *$ \\
\hline F2 & & - & $16^{\mathrm{ns}}$ & $45^{*}$ \\
\hline F3 & & & - & $29^{\text {ns }}$ \\
\hline $\mathrm{F} 4$ & & & & - \\
\hline
\end{tabular}
mínima significativa= 34. F1 (70\% M: 30\% AB), F2 (60\% M: 40\% AB), F3 (40\% M: 60\% AB) e F4 (30\% M: 70\% AB). 


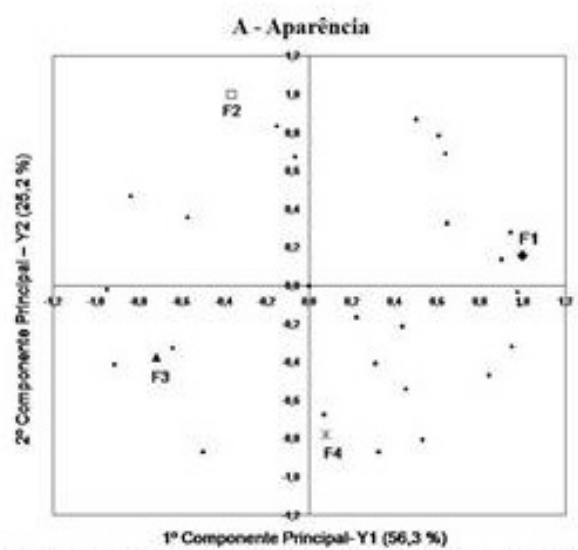

$F 1-70 \%, M-30 \times, A B ; F 2-60 \%$ M-40\%, $A B ; M ; F 3-40 \%$ M.60\% $A B ; F 4-30 \%, M-70 \%$ AB

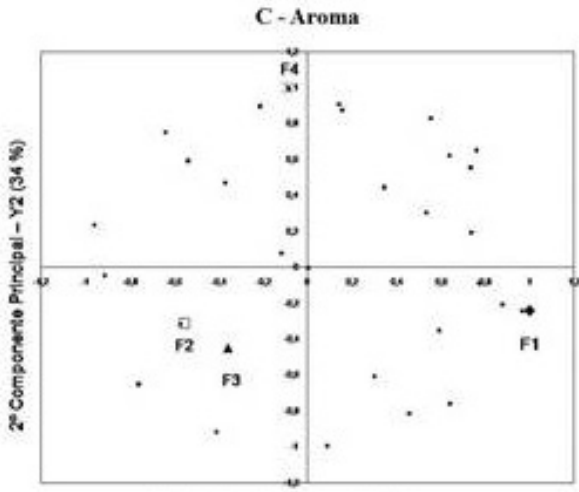

10 Componente Principat-Y1 (40 \$)

F1- 70\% M:30 \% NB; F2- $60 \%$ M:40\%, AB; M; F3-40\% M.60\% NB; F4- 30\% M: $70 \%$ NB

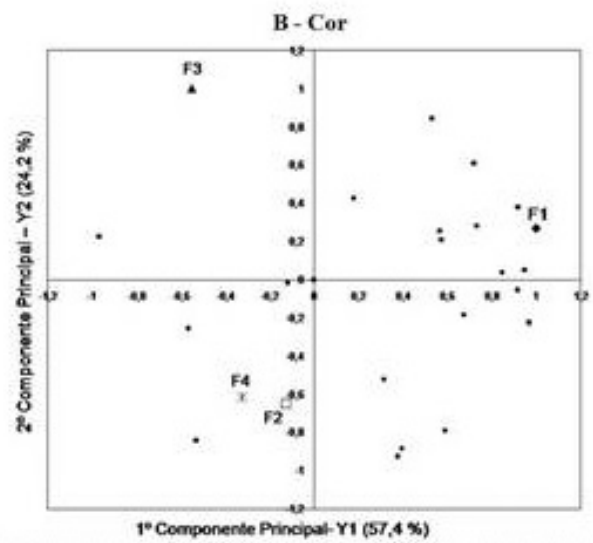

F1- 70\%, M30 \% NB; F2- 60\%, M-40\% AB; M; F3-40\%, ME0\%, AB; F4- 30\% M-70\%, AB

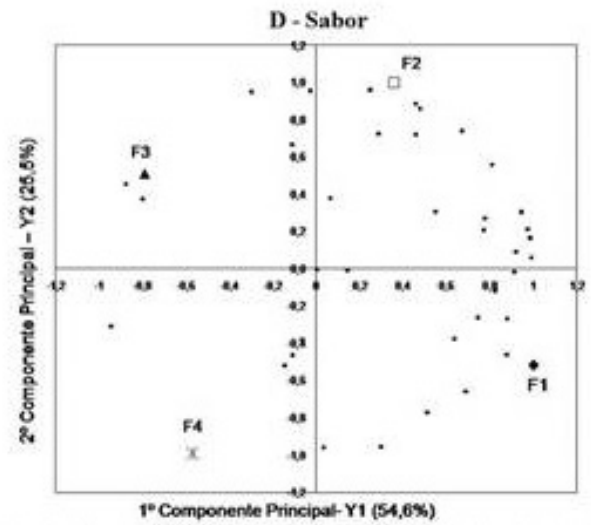

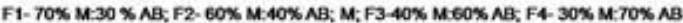

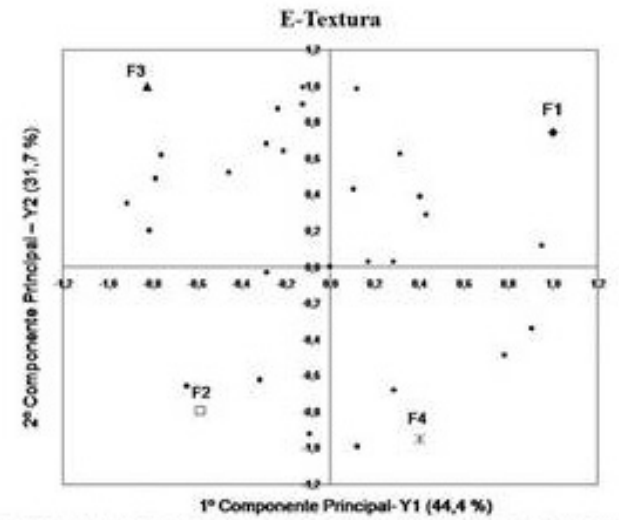

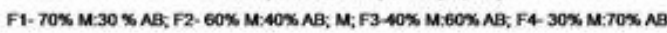

FIGURA 3- Mapa de Preferência Interno para as quatro formulações de geleia de mamão (M) e araçá-boi $(\mathrm{AB})$ em relação aos atributos sensoriais avaliados. 


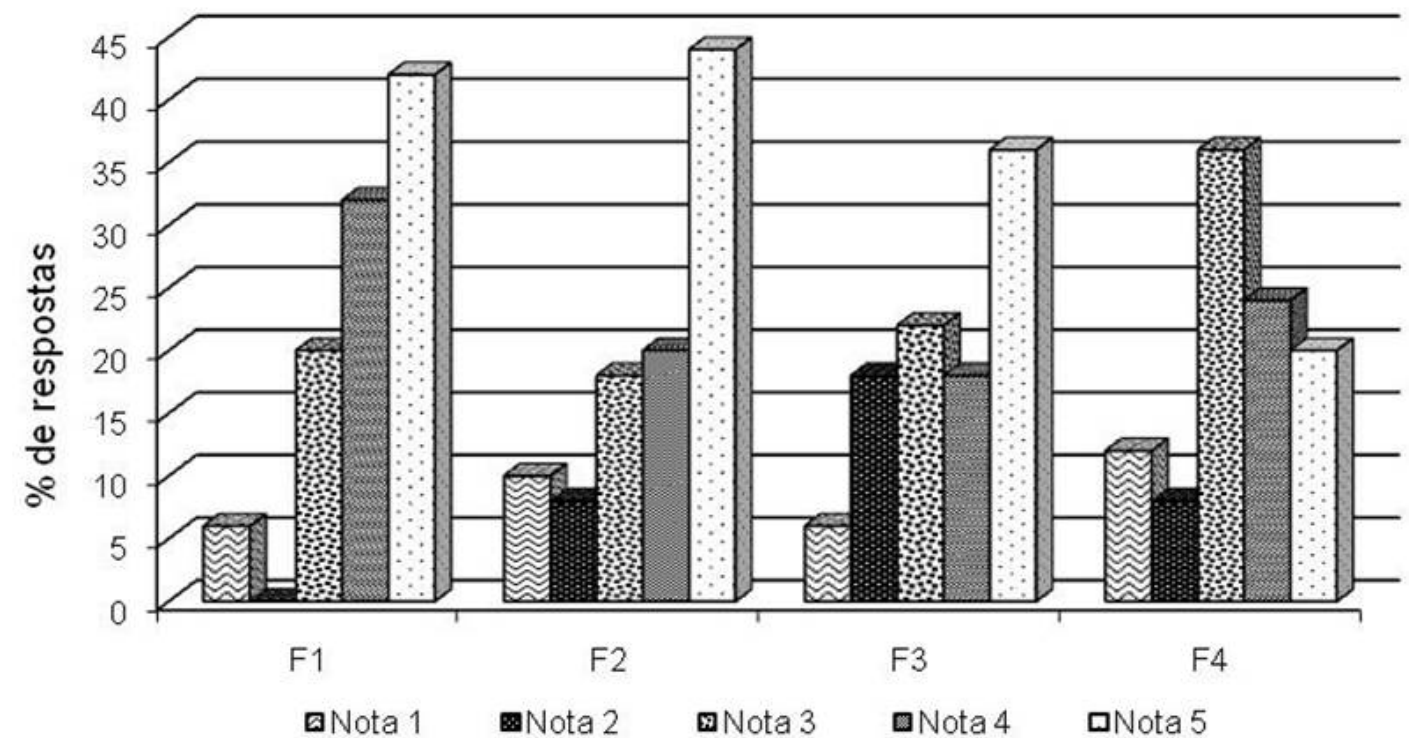

FIGURA 4- Intenção de compra de geleias elaboradas com mamão (M) + araçá-boi (AB) em diferentes proporções. Nota 1- certamente não compraria; Nota 2- possivelmente não compraria; Nota 3talvez comprasse/talvez não comprasse; Nota 4-possivelmente compraria; Nota 5- certamente compraria. Formulações: F1 (70\% M: 30\% AB), F2 (60\% M: 40\% AB), F3 (40\% M: 60\% $\mathrm{AB})$ e F4 (30\% M: $70 \% \mathrm{AB})$.

\section{CONCLUSÃO}

A formulação de geleia contendo $70 \%$ de mamão e $30 \%$ de araçá-boi apresenta maior aceitação em relação aos atributos sabor, cor e aparência, e elevada intenção de compra pelos consumidores $(74 \%)$, indicando ser adequada para a industrialização e consumo.

A análise dos dados, utilizando-se do Mapa de Preferência Interno, mostra-se mais eficiente do que a análise de variância e a análise de frequência, retratando com maior fidelidade a percepção sensorial de cada consumidor em relação ao produto.

\section{AGRADECIMENTOS}

Os autores agradecem à Fundação de Amparo à Pesquisa do Estado da Bahia (FAPESB), pelo apoio financeiro a este projeto.

\section{REFERÊNCIAS}

BASU, S.; SHIVHRARE, U.S.; SINGH, T.V.; BENIWAL, V.S. Rheological, textural and spectral characteristics of sorbitol substituted mango jam. Journal of Food Engineering, Oxford, v.105, p.503-512, 2011.
FERREIRA, R.M.A.; AROUCHA, E.M.M.; GÓIS, V.A.; SILVA, D.K.; SOUSA, C.M.G. Qualidade sensorial de geleia mista de melancia e tamarindo. Revista Caatinga, Mossoró, v.24, n.2, p.202-206, 2011.

IAL - Instituto Adolfo Lutz. Métodos físicoquímicos para análise de alimentos. 4. ed. Brasília: Ministério da Saúde, 2005. 1018p.

LAGO, E. S.; GOMES, E.; SILVA, R. Produção de geleia de jambolão (Syzygium cumini lamarck): processamento, parâmetros físico-químicos e avaliação sensorial. Ciência e Tecnologia de Alimentos, Campinas, v.26, n.4 p.847-852, 2006.

LAGO-VANZELA, E. S. RAMIN, P.; GUEZUMSZA, M. A.; SAMTPS, G. V.; GOMES, E. DA SILVA, R. Chemical and sensory characteristics of pulp and peel cajá-manga (Spondias cytherea Sonn.) jelly. Ciência e Tecnologia de Alimentos, Campinas, v. 31, n.2, p.398-405, 2011.

MARTÍN-ESPARZA, M. E.; ESCRICHE, I.; PENAGOS, L.; MATÍNEZ-NAVARRETE, N. Quality stability assessment of a strawberry-gel product during storage. Journal of Food Process Engineering, London, v.34, p.204-223, 2011a. 
MARTÍN-ESPARZA, M. E.; ESCRICHE, I.; PENAGOS, L.; MATÍNEZ-NAVARRETE, N. Significance of osmotic temperature treatment and storage time on physical and chemical properties of a strawberr-gel product. Journal of Science and Food Agriculture, Chichester, v.91, p.894-904, 2011 b.

MOTA, R. V. Caracterização física e química de geleia de amora-preta. Ciência e Tecnologia de Alimentos, Campinas, v.26, n.3. p.539-543. 2006.

MOTA, R. V. Características químicas e aceitabilidade de geleias de amora-preta de baixo teor de sólidos solúveis. Brazilian Journal of Food Technology, Campinas, v.10, n.2, p.116-121, 2007.

NELSON, N. A photometric adaptation of the Somogyi method for the determination of glucose. The Journal of Biological Chemistry, Baltimore, v.153, n.1, p.375-380, 1944.

SACRAMENTO, C. K.; BARRETTO, W. S.; FARIA, J. C. Araçá-boi: uma alternativa para agroindústria. Bahia Agrícola, Salvador, v.8, p.22-24, 2008.
SERRANO, L.A.L.; CATTANEO, L.F. O cultivo do mamoeiro no Brasil. Revista Brasileira de Fruticultura, Jaboticabal, v.32, n.3, 2010.

SINGH, S.; JAIN, S.; SINGH, S. P.; SINGH, D. Quality changes in fruit jams from combinations of different fruit pulps. Journal of Food Processing and Preservation, Hoboken, v.33, p.41-47, 2009.

SOMOGYI, M. A new reagent for the determination of sugar. The Journal of Biological Chemistry, Baltimore, v.160, n.1, p.61-68, set., 1945.

STONE, H.; SIDEL, J. L. Sensory evaluation practices. $3^{\text {rd }}$ ed. London: Academic Press, 2004. $408 p$.

YUYAMA, L. K. O. PANTOJA, L., MAEDA, R.N., AGUIAR, J.P.L., SILVA, J.P.L.A. Desenvolvimento e aceitabilidade de geléia dietética de cubiu (Solanum sessiliflorum Dunal). Ciência e Tecnologia de Alimentos, Campinas, v.28, n.4, p.929-934., 2008. 